\title{
Some inequalities obtained by fractional integrals of positive real orders
}

\author{
Mustafa Gürbüz ${ }^{1 *}$ (D), Yakup Taşdan² and Erhan Set $^{3}$
}

\section{"Correspondence:}

mgurbuz@agri.edu.t

'Department of Mathematics,

Education Faculty, Ağrı İbrahim

Çeçen University, Ağrı, Turkey

Full list of author information is

available at the end of the article

\section{Springer}

\begin{abstract}
The primary objective of this study is to handle new generalized Hermite-Hadamard type inequalities with the help of the Katugampola fractional integral operator, which generalizes the Hadamard and Riemann-Liouville fractional integral operators into one system. In order to do this, a new fractional integral identity is obtained. Then, by using this identity, some inequalities for the class of functions whose derivatives in absolute values at certain powers are $\rho$-convex are derived. It is observed that the obtained inequalities are generalizations of some results in the literature.
\end{abstract}

MSC: 26A33; 26D10; 26D15

Keywords: Katugampola fractional integrals; Hadamard type inequalities; $\rho$-convex functions

\section{Introduction}

It is a well known fact that inequalities have important roles to play in the studies of linear programming, extremum problems, optimization, error estimates and game theory (see for example [2]). Over the years, only integer real order integrals were taken into account while handling new results about integral inequalities. However, in recent years, fractional calculus has been considered by many scientists (see $[3-5,7-10,12,13])$. There are some inequalities in the literature that accelerated studies on integral inequalities. One of the most famous and practical inequalities in the literature was the Hermite-Hadamard inequality given in the following theorem.

Theorem 1.1 Let $f$ be defined from interval I (a nonempty subset of $\mathbb{R}$ ) to $\mathbb{R}$ to be a convex function on I and $a, b \in I$ with $a<b$. Then the double inequality given in the following holds:

$$
f\left(\frac{a+b}{2}\right) \leq \frac{1}{b-a} \int_{a}^{b} f(x) d x \leq \frac{f(a)+f(b)}{2} .
$$

Zhang and Wan introduced $p$-convex functions in [13] and İșcan gave a different version of this definition in [3] as follows.

(c) The Author(s) 2020. This article is licensed under a Creative Commons Attribution 4.0 International License, which permits use sharing, adaptation, distribution and reproduction in any medium or format, as long as you give appropriate credit to the original author(s) and the source, provide a link to the Creative Commons licence, and indicate if changes were made. The images or other third party material in this article are included in the article's Creative Commons licence, unless indicated otherwise in a credit line to the material. If material is not included in the article's Creative Commons licence and your intended use is not permitted by statutory regulation or exceeds the permitted use, you will need to obtain permission directly from the copyright holder. To view a copy of this licence, visit http://creativecommons.org/licenses/by/4.0/. 
Definition 1.1 Let $I$ be an interval composed of positive real numbers and $p \in \mathbb{R} \backslash\{0\}$. $f: I \rightarrow \mathbb{R}$ is called a $p$-convex function if it satisfies

$$
f\left(\left[t x^{p}+(1-t) y^{p}\right]^{\frac{1}{p}}\right) \leq t f(x)+(1-t) f(y)
$$

for all $t \in[0,1]$ and $x, y \in I$.

It is easy to see that ordinary convexity is retrieved from $p$-convexity for $p=1$ and harmonically convexity is retrieved from $p$-convexity for $p=-1$.

Now we will mention some kinds of fractional integral operators and the definition in the space $X_{c}^{p}(a, b)$.

The first of them is the Riemann-Liouville fractional integral, which makes the integration of fractional order possible (see [9]).

Definition 1.2 Let $f \in L_{1}[a, b] . J_{a+}^{\alpha} f$ and $J_{b-}^{\alpha} f$, which are called left-sided and right-sided Riemann-Liouville integrals of order $\alpha>0$ with $a \geq 0$, are defined by

$$
J_{a+}^{\alpha} f=\frac{1}{\Gamma(\alpha)} \int_{a}^{x}(x-t)^{\alpha-1} f(t) d t, \quad x>a,
$$

and

$$
J_{b-}^{\alpha} f=\frac{1}{\Gamma(\alpha)} \int_{x}^{b}(t-x)^{\alpha-1} f(t) d t, \quad x<b,
$$

respectively, where $\Gamma(\alpha)=\int_{0}^{\infty} e^{-t} u^{\alpha-1} d u$. Here $J_{a^{+}}^{0} f(x)=J_{b^{-}}^{0} f(x)=f(x)$.

Definition 1.3 ([9]) The left-sided and right-sided Hadamard fractional integrals of order $\alpha \in \mathbb{R}^{\alpha}$ are defined as

$$
\begin{array}{ll}
H_{a+}^{\alpha} \varphi=\frac{1}{\Gamma(\alpha)} \int_{a}^{x} \frac{\varphi(t)}{\left(\ln \frac{x}{t}\right)^{1-\alpha}} \frac{d t}{t}, \quad x>a>0, \\
H_{b-}^{\alpha} \varphi=\frac{1}{\Gamma(\alpha)} \int_{x}^{b} \frac{\varphi(t)}{\left(\ln \frac{t}{x}\right)^{1-\alpha}} \frac{d t}{t}, \quad 0<x<b,
\end{array}
$$

where $\Gamma$ is the gamma function.

Definition 1.4 ([8]) Let us consider the space $X_{c}^{p}(a, b)(1 \leq p \leq \infty, c \in \mathbb{R})$ of the Lebesque measurable complex-valued mappings $f$ on $[a, b]$ which satisfy $\|f\| x_{c}^{p}<\infty$ where the norm is defined for the case $1 \leq p \leq \infty, c \in \mathbb{R}$ as follows:

$$
\|f\| x_{c}^{p}-\left(\int_{a}^{b}\left|t^{c} f(t)\right|^{p} \frac{d t}{t}\right)^{\frac{1}{p}}<\infty
$$

and, for the case $p=\infty$,

$$
\|f\| x_{c}^{p}=\operatorname{ess} \sup _{a \leq t \leq b}\left[t^{c}|f(t)|\right] \quad(c \in \mathbb{R}) .
$$


Katugampola revealed a new fractional integration operator which generalizes both the Riemann-Liouville and the Hadamard fractional integration operators. This integration operator possesses the semigroup properties (see $[4,5])$ and is defined as follows.

Definition 1.5 Let $[a, b] \subset \mathbb{R}$ be a finite interval. Then, the left- and right-side Katugampola fractional integrals of order $(\alpha>0)$ of $f \in X_{c}^{p}(a, b)$ are defined by

$$
{ }^{\rho} I_{a+}^{\alpha} f(x)=\frac{\rho^{1-\alpha}}{\Gamma(\alpha)} \int_{a}^{x} \frac{t^{\rho-1}}{\left(x^{\rho}-t^{\rho}\right)^{1-\alpha}} f(t) d t
$$

and

$$
{ }^{\rho} I_{b-}^{\alpha} f(x)=\frac{\rho^{1-\alpha}}{\Gamma(\alpha)} \int_{x}^{b} \frac{t^{\rho-1}}{\left(t^{\rho}-x^{\rho}\right)^{1-\alpha}} f(t) d t
$$

with $a<x<b$ and $\rho>0$ if the integral exists. Equations (9) and (10) look quite the same as the Erdelyi-Kober operator. But besides the Hadamard fractional integrals not being a direct consequence of the Erdelyi-Kober operator, they are a direct consequence of the Katugampola fractional integral operators.

Theorem $1.2([5])$ Let $\alpha>0$ and $\rho>0$. Then, for $x>a$,

$$
\begin{aligned}
& \text { 1. } \lim _{\rho \rightarrow 1}{ }^{\rho} I_{a+}^{\alpha} f(x)=J_{a_{+}}^{\alpha} f(x), \\
& \text { 2. } \lim _{\rho \rightarrow 0^{+}}{ }^{\rho} I_{a+}^{\alpha} f(x)=H_{a^{+}}^{\alpha} f(x) .
\end{aligned}
$$

For right-sided operators, a similar conclusion can be drawn.

For more studies of fractional integral inequalities, see [10,12] and the references therein.

Erdelyi et al. were deeply involved in hypergeometric functions given in the following (see [1]):

$$
{ }_{2} F_{1}(a, b ; c ; z)=\frac{1}{\beta(b, b-c)} \int_{0}^{1} t^{b-1}(1-t)^{c-b-1}(1-z t)^{-a} d t, \quad c>b>0,|z|<1
$$

and the regularized hypergeometric function is

$$
{ }_{p} \widetilde{F}_{q}\left[a_{1}, \ldots, a_{p} ; b_{1}, \ldots, b_{q} ; z\right] \equiv \frac{{ }_{p} F_{q}\left[a_{1}, \ldots, a_{p} ; b_{1}, \ldots, b_{q} ; z\right]}{\Gamma\left(b_{1}\right) \cdots \Gamma\left(b_{q}\right)}
$$

given in [11]. We will define $T_{f}(\alpha, \rho ; a, x, b)$ by

$$
\begin{aligned}
T_{f}(\alpha, \rho ; a, x, b)= & \frac{\rho}{b-a}\left[\left(x^{\rho}-a^{\rho}\right)^{\alpha} f(a)+\left(b^{\rho}-x^{\rho}\right)^{\alpha} f(b)\right] \\
& -\frac{\rho^{\alpha+1} \Gamma(\alpha+1)}{b-a}\left[{ }^{\rho} I_{x^{-}}^{\alpha} f(a)+{ }^{\rho} I_{x^{+}}^{\alpha} f(b)\right]
\end{aligned}
$$

and $\Gamma$ is the Euler Gamma function, i.e., $\Gamma(\alpha)=\int_{0}^{\infty} e^{-u} u^{\alpha-1} d u$.

Kavurmacı et al. obtained new Ostrowski type results after proving the next lemma in 2011 in [6]. 
Lemma 1.1 ([6]) Let $f$ be defined from an interval I to $\mathbb{R}$ as a differentiable mapping on the interior of $I$, where $a, b \in I, a<b$ and $f^{\prime} \in L[a, b]$. Then the equality given here is valid:

$$
\begin{gathered}
\frac{(x-a) f(a)+(b-x) f(b)}{b-a}-\frac{1}{b-a} \int_{a}^{b} f(x) d x \\
=\frac{(x-a)^{2}}{b-a} \int_{0}^{1}(t-1) f^{\prime}(t x+(1-t) a) d t \\
+\frac{(b-x)^{2}}{b-a} \int_{0}^{1}(1-t) f^{\prime}(t x+(1-t) b) d t .
\end{gathered}
$$

Kavurmacı et al. presented the next lemma to handle Ostrowski type inequalities for Riemann-Liouville fractional integrals in 2012 in [7].

Lemma 1.2 ([7]) Let $f$ be defined from interval I to $\mathbb{R}$ as a differentiable function on $I^{\circ}$, where $a$ and $b$ belong to $I$ with $a<b$ and $f^{\prime} \in L[a, b]$. Then we get

$$
\begin{gathered}
\frac{(x-a)^{\alpha} f(a)+(b-x)^{\alpha} f(b)}{b-a}-\frac{\Gamma(\alpha+1)}{b-a}\left[J_{x}^{\alpha} f(a)+J_{x^{+}}^{\alpha} f(b)\right] \\
=\frac{(x-a)^{\alpha+1}}{b-a} \int_{0}^{1}\left(t^{\alpha}-1\right) f^{\prime}(t x+(1-t) a) d t \\
\quad+\frac{(b-x)^{\alpha+1}}{b-a} \int_{0}^{1}\left(1-t^{\alpha}\right) f^{\prime}(t x+(1-t) b) d t
\end{gathered}
$$

for all $x \in[a, b]$ and $\alpha>0$.

In this paper, a new kernel and Ostrowski type new theorems including the Katugampola fractional integral operator have been retrieved inspired by Lemma 1.2.

\section{Main results}

Lemma 2.1 Let $f$ be defined from interval I which consists of positive real numbers to $\mathbb{R}$ as a differentiable function on $I^{\circ}$, where $a, b \in I$ with $a<b$ and $f^{\prime} \in L[a, b]$. Then we have

$$
\begin{aligned}
T_{f}(\alpha, \rho ; a, x, b)= & \frac{\left(x^{\rho}-a^{\rho}\right)^{\alpha+1}}{b-a} \int_{0}^{1} \frac{\left(t^{\alpha}-1\right) f^{\prime}\left(\left[t x^{\rho}+(1-t) a^{\rho}\right]^{\frac{1}{\rho}}\right)}{\left(t x^{\rho}+(1-t) a^{\rho}\right)^{1-\frac{1}{\rho}}} d t \\
& +\frac{\left(b^{\rho}-x^{\rho}\right)^{\alpha+1}}{b-a} \int_{0}^{1} \frac{\left(1-t^{\alpha}\right) f^{\prime}\left(\left[t x^{\rho}+(1-t) b^{\rho}\right]^{\frac{1}{\rho}}\right)}{\left(t x^{\rho}+(1-t) b^{\rho}\right)^{1-\frac{1}{\rho}}} d t
\end{aligned}
$$

for all $x \in[a, b], \rho>0$ and $\alpha>0$.

Proof With the help of partial integration we have

$$
\begin{aligned}
I_{1} & =\int_{0}^{1} \frac{\left(t^{\alpha}-1\right) f^{\prime}\left(\left[t x^{\rho}+(1-t) a^{\rho}\right]^{\frac{1}{\rho}}\right)}{\left(t x^{\rho}+(1-t) a^{\rho}\right)^{1-\frac{1}{\rho}}} d t \\
& =\frac{\rho f(a)}{x^{\rho}-a^{\rho}}-\frac{\alpha \rho}{x^{\rho}-a^{\rho}} \int_{0}^{1} t^{\alpha-1} f\left(\left[t x^{\rho}+(1-t) a^{\rho}\right]^{\frac{1}{\rho}}\right) d t .
\end{aligned}
$$


By changing the variable $\left[t x^{\rho}+(1-t) a^{\rho}\right]^{\frac{1}{\rho}}=u$ we get

$$
\begin{aligned}
I_{1} & =\frac{\rho f(a)}{x^{\rho}-a^{\rho}}-\frac{\alpha \rho}{x^{\rho}-a^{\rho}} \int_{a}^{x}\left(\frac{u^{\rho}-a^{\rho}}{x^{\rho}-a^{\rho}}\right)^{\alpha-1} \frac{\rho u^{\rho-1}}{x^{\rho}-a^{\rho}} f(u) d u \\
& =\frac{\rho f(a)}{x^{\rho}-a^{\rho}}-\frac{\alpha \rho^{2}}{\left(x^{\rho}-a^{\rho}\right)^{\alpha+1}} \int_{a}^{x} \frac{u^{\rho-1}}{\left(u^{\rho}-a^{\rho}\right)^{1-\alpha}} f(u) d u \\
& =\frac{\rho f(a)}{x^{\rho}-a^{\rho}}-\frac{\alpha \rho^{2} \Gamma(\alpha)}{\left(x^{\rho}-a^{\rho}\right)^{\alpha+1} \rho^{1-\alpha}} I_{x^{-}}^{\alpha} f(a) \\
& =\frac{\rho f(a)}{x^{\rho}-a^{\rho}}-\frac{\rho^{\alpha+1} \Gamma(\alpha+1)}{\left(x^{\rho}-a^{\rho}\right)^{\alpha+1}} I_{x^{-}}^{\alpha} f(a) .
\end{aligned}
$$

Similarly we have

$$
\begin{aligned}
I_{2}= & \int_{0}^{1} \frac{\left(1-t^{\alpha}\right) f^{\prime}\left(\left[t x^{\rho}+(1-t) b^{\rho}\right]^{\frac{1}{\rho}}\right)}{\left(t x^{\rho}+(1-t) b^{\rho}\right)^{1-\frac{1}{\rho}}} d t \\
I_{2}= & \left.\frac{\rho\left(1-t^{\alpha}\right)}{x^{\rho}-b^{\rho}} f\left(\left[t x^{\rho}+(1-t) b^{\rho}\right]^{\frac{1}{\rho}}\right)\right|_{0} ^{1} \\
& +\frac{\alpha \rho}{x^{\rho}-b^{\rho}} \int_{0}^{1} t^{\alpha-1} f\left(\left[t x^{\rho}+(1-t) b^{\rho}\right]^{\frac{1}{\rho}}\right) d t \\
= & \frac{\rho f(b)}{b^{\rho}-x^{\rho}}-\frac{\alpha \rho}{b^{\rho}-x^{\rho}} \int_{0}^{1} t^{\alpha-1} f\left(\left[t x^{\rho}+(1-t) b^{\rho}\right]^{\frac{1}{\rho}}\right) d t .
\end{aligned}
$$

By changing the variable $\left[t x^{\rho}+(1-t) b^{\rho}\right]^{\frac{1}{\rho}}=u$ we get

$$
\begin{aligned}
I_{2} & =\frac{\rho f(b)}{b^{\rho}-x^{\rho}}-\frac{\alpha \rho}{b^{\rho}-x^{\rho}} \int_{b}^{x}\left(\frac{u^{\rho}-b^{\rho}}{x^{\rho}-b^{\rho}}\right)^{\alpha-1} \frac{\rho u^{\rho-1}}{x^{\rho}-b^{\rho}} f(u) d u \\
& =\frac{\rho f(b)}{b^{\rho}-x^{\rho}}-\frac{\alpha \rho^{2}}{\left(b^{\rho}-x^{\rho}\right)^{\alpha+1}} \int_{x}^{b} \frac{u^{\rho-1}}{\left(b^{\rho}-u^{\rho}\right)^{1-\alpha}} f(u) d u \\
& =\frac{\rho f(b)}{b^{\rho}-x^{\rho}}-\frac{\alpha \rho^{2} \Gamma(\alpha)}{\left(b^{\rho}-x^{\rho}\right)^{\alpha+1} \rho^{1-\alpha}}{ }^{\rho} I_{x^{+}}^{\alpha} f(b) \\
& =\frac{\rho f(b)}{b^{\rho}-x^{\rho}}-\frac{\rho^{\alpha+1} \Gamma(\alpha+1)}{\left(b^{\rho}-x^{\rho}\right)^{\alpha+1}} I_{x^{+}}^{\alpha} f(b) .
\end{aligned}
$$

By multiplying (20) and (23) with $\frac{\left(x^{\rho}-a^{\rho}\right)^{\alpha+1}}{b-a}$ and $\frac{\left(b^{\rho}-x^{\rho}\right)^{\alpha+1}}{b-a}$, respectively, and then summing them side by side, we have

$$
\begin{gathered}
\frac{\left(x^{\rho}-a^{\rho}\right)^{\alpha+1}}{b-a} \int_{0}^{1} \frac{\left(t^{\alpha}-1\right) f^{\prime}\left(\left[t x^{\rho}+(1-t) a^{\rho}\right]^{\frac{1}{\rho}}\right)}{\left(t x^{\rho}+(1-t) a^{\rho}\right)^{1-\frac{1}{\rho}}} d t \\
\quad+\frac{\left(b^{\rho}-x^{\rho}\right)^{\alpha+1}}{b-a} \int_{0}^{1} \frac{\left(1-t^{\alpha}\right) f^{\prime}\left(\left[t x^{\rho}+(1-t) b^{\rho}\right]^{\frac{1}{\rho}}\right)}{\left(t x^{\rho}+(1-t) b^{\rho}\right)^{1-\frac{1}{\rho}}} d t \\
=\frac{\rho f(a)\left(x^{\rho}-a^{\rho}\right)^{\alpha}}{b-a}-\frac{\rho^{\alpha+1} \Gamma(\alpha+1)^{\rho} I_{x^{-}}^{\alpha} f(a)}{b-a} \\
+\frac{\rho f(b)\left(b^{\rho}-x^{\rho}\right)^{\alpha}}{b-a}-\frac{\rho^{\alpha+1} \Gamma(\alpha+1)^{\rho} I_{x^{+}}^{\alpha} f(b)}{b-a} .
\end{gathered}
$$

By rearranging the last equality we get the desired equality. 
Remark 2.1 If we choose $\rho \rightarrow 1$ in Lemma 2.1, we get Lemma 1.2 proved in [7].

Remark 2.2 By choosing $\rho \rightarrow 1$ and $\alpha=1$ in Lemma 2.1, we get Lemma 1.1 proved in [6].

Theorem 2.1 Let $f$ be defined from interval I which consists of positive real numbers to $\mathbb{R}$ as a differentiable mapping on $I^{\circ}$ and $a, b \in I$ with $a<b$ such that $f^{\prime} \in L[a, b]$. If $\left|f^{\prime}\right|$ is $\rho$-convex on I we have

$$
\begin{aligned}
\left|T_{f}(\alpha, \rho ; a, x, b)\right| \leq & \frac{\left(x^{\rho}-a^{\rho}\right)^{\alpha+1}}{b-a}\left\{\left|f^{\prime}(x)\right| K(a)+\left|f^{\prime}(a)\right| L(a)\right\} \\
& +\frac{\left(b^{\rho}-x^{\rho}\right)^{\alpha+1}}{b-a}\left\{\left|f^{\prime}(x)\right| K(b)+\left|f^{\prime}(b)\right| L(b)\right\}
\end{aligned}
$$

where

$$
\begin{aligned}
K(a)= & \frac{\rho\left(x^{1+\rho}-a^{\rho}(-a \rho+x+\rho x)\right)}{(1+\rho)\left(x^{\rho}-a^{\rho}\right)^{2}} \\
& -a^{1-\rho} \Gamma(2+\alpha)_{1} \widetilde{F}_{2}\left(2+\alpha, \frac{\rho-1}{\rho} ; 3+\alpha ; 1-\left(\frac{x}{a}\right)^{\rho}\right), \\
K(b)= & \frac{\rho\left(x^{1+\rho}-b^{\rho}(-b \rho+x+\rho x)\right)}{(1+\rho)\left(x^{\rho}-b^{\rho}\right)^{2}} \\
& -b^{1-\rho} \Gamma(2+\alpha) \widetilde{F}_{2}\left(2+\alpha, \frac{\rho-1}{\rho} ; 3+\alpha ; 1-\left(\frac{x}{b}\right)^{\rho}\right), \\
L(a)= & \frac{\left(t x^{\rho}+a^{\rho}\right)^{\frac{1-\rho}{\rho}}\left[{ }_{2} F_{1}\left(1, p-1 ; p+2 ; \frac{a^{\rho}}{t x^{\rho}+a^{\rho}}\right)\right]^{\frac{1}{\rho}}}{(\rho+1)^{\frac{1}{\rho}}}\left(\frac{\Gamma(q+1) \Gamma\left(1+\frac{1}{\alpha}\right)}{\Gamma\left(1+q+\frac{1}{\alpha}\right)}\right)^{\frac{1}{q}}, \\
L(b)= & \frac{\left(t x^{\rho}+b^{\rho}\right)^{\frac{1-\rho}{\rho}}\left[{ }_{2} F_{1}\left(1, p-1 ; p+2 ; \frac{b^{\rho}}{t x^{\rho}+b^{\rho}}\right)\right]^{\frac{1}{\rho}}}{(\rho+1)^{\frac{1}{\rho}}}\left(\frac{\Gamma(q+1) \Gamma\left(1+\frac{1}{\alpha}\right)}{\Gamma\left(1+q+\frac{1}{\alpha}\right)}\right)^{\frac{1}{q}},
\end{aligned}
$$

and for all $x \in\left(a, 2^{\frac{1}{\rho}} a\right)\left(\right.$ if $2^{\frac{1}{\rho}} a<b$, otherwise $\left.x \in(a, b)\right), \alpha>0, \rho>1, q>1$ and $\frac{1}{\rho}+\frac{1}{q}=1$.

Proof Using Lemma 2.1 and the properties of the absolute value we get

$$
\begin{aligned}
\left|T_{f}(\alpha, \rho ; a, x, b)\right| \leq & \frac{\left(x^{\rho}-a^{\rho}\right)^{\alpha+1}}{b-a} \int_{0}^{1} \frac{\left(1-t^{\alpha}\right)\left|f^{\prime}\left(\left[t x^{\rho}+(1-t) a^{\rho}\right]^{\frac{1}{\rho}}\right)\right|}{\left(t x^{\rho}+(1-t) a^{\rho}\right)^{1-\frac{1}{\rho}}} d t \\
& +\frac{\left(b^{\rho}-x^{\rho}\right)^{\alpha+1}}{b-a} \int_{0}^{1} \frac{\left(1-t^{\alpha}\right)\left|f^{\prime}\left(\left[t x^{\rho}+(1-t) b^{\rho}\right]^{\frac{1}{\rho}}\right)\right|}{\left(t x^{\rho}+(1-t) b^{\rho}\right)^{1-\frac{1}{\rho}}} d t .
\end{aligned}
$$

Then by taking into account the $\rho$-convexity of $\left|f^{\prime}\right|$ and the Hölder inequality we get

$$
\begin{aligned}
& T_{f}(\alpha, \rho ; a, x, b) \\
& \leq \frac{\left(x^{\rho}-a^{\rho}\right)^{\alpha+1}}{b-a}\left\{\int_{0}^{1} \frac{\left(t-t^{\alpha+1}\right)\left|f^{\prime}(x)\right|}{\left(t x^{\rho}+(1-t) a^{\rho}\right)^{1-\frac{1}{\rho}}} d t+\int_{0}^{1} \frac{(1-t)\left(1-t^{\alpha}\right)\left|f^{\prime}(a)\right|}{\left(t x^{\rho}+(1-t) a^{\rho}\right)^{1-\frac{1}{\rho}}} d t\right\} \\
& \quad+\frac{\left(b^{\rho}-x^{\rho}\right)^{\alpha+1}}{b-a}\left\{\int_{0}^{1} \frac{\left(t-t^{\alpha+1}\right)\left|f^{\prime}(x)\right|}{\left(t x^{\rho}+(1-t) b^{\rho}\right)^{1-\frac{1}{\rho}}} d t+\int_{0}^{1} \frac{(1-t)\left(1-t^{\alpha}\right)\left|f^{\prime}(b)\right|}{\left(t x^{\rho}+(1-t) b^{\rho}\right)^{1-\frac{1}{\rho}}} d t\right\}
\end{aligned}
$$




$$
\begin{aligned}
\leq & \frac{\left(x^{\rho}-a^{\rho}\right)^{\alpha+1}}{b-a}\left\{\int_{0}^{1} \frac{\left(t-t^{\alpha+1}\right)\left|f^{\prime}(x)\right|}{\left(t x^{\rho}+(1-t) a^{\rho}\right)^{1-\frac{1}{\rho}}} d t\right. \\
& \left.+\left(\int_{0}^{1} \frac{(1-t)^{\rho}}{\left(t x^{\rho}+(1-t) a^{\rho}\right)^{\rho-1}} d t\right)^{\frac{1}{\rho}}\left(\int_{0}^{1}\left(1-t^{\alpha}\right)^{q} d t\right)^{\frac{1}{q}}\left|f^{\prime}(a)\right|\right\} \\
& +\frac{\left(b^{\rho}-x^{\rho}\right)^{\alpha+1}}{b-a}\left\{\int_{0}^{1} \frac{\left(t-t^{\alpha+1}\right)\left|f^{\prime}(x)\right|}{\left(t x^{\rho}+(1-t) b^{\rho}\right)^{1-\frac{1}{\rho}}} d t\right. \\
& \left.+\left(\int_{0}^{1} \frac{(1-t)^{\rho}}{\left(t x^{\rho}+(1-t) b^{\rho}\right)^{\rho-1}} d t\right)^{\frac{1}{\rho}}\left(\int_{0}^{1}\left(1-t^{\alpha}\right)^{q} d t\right)^{\frac{1}{q}}\left|f^{\prime}(b)\right|\right\}
\end{aligned}
$$

By the necessary computations we have

$$
\begin{aligned}
& K(a)=\int_{0}^{1} \frac{\left(t-t^{\alpha+1}\right)}{\left(t x^{\rho}+(1-t) a^{\rho}\right)^{1-\frac{1}{\rho}}} d t, \\
& K(b)=\int_{0}^{1} \frac{\left(t-t^{\alpha+1}\right)}{\left(t x^{\rho}+(1-t) b^{\rho}\right)^{1-\frac{1}{\rho}}} d t, \\
& L(a)=\left(\int_{0}^{1} \frac{(1-t)^{\rho}}{\left(t x^{\rho}+(1-t) a^{\rho}\right)^{\rho-1}} d t\right)^{\frac{1}{\rho}}\left(\int_{0}^{1}\left(1-t^{\alpha}\right)^{q} d t\right)^{\frac{1}{q}}, \\
& L(b)=\left(\int_{0}^{1} \frac{(1-t)^{\rho}}{\left(t x^{\rho}+(1-t) b^{\rho}\right)^{\rho-1}} d t\right)^{\frac{1}{\rho}}\left(\int_{0}^{1}\left(1-t^{\alpha}\right)^{q} d t\right)^{\frac{1}{q}},
\end{aligned}
$$

where $K(a), K(b), L(a)$ and $L(b)$ are defined as in (26), (27), (28) and (29), respectively. So the proof is completed.

Theorem 2.2 Let $f$ be defined from an interval I which consists of positive real numbers to $\mathbb{R}$ as a differentiable mapping on $I^{\circ}$ and $a, b \in I$ with $a<b$ such that $f^{\prime} \in L[a, b]$. If $\left|f^{\prime}\right|^{q}$ is $\rho$-convex on I we have

$$
\begin{aligned}
& \left|T_{f}(\alpha, \rho ; a, x, b)\right| \\
& \leq \frac{\left(x^{\rho}-a^{\rho}\right)^{\alpha+1}}{b-a} M^{\frac{1}{r}}(a)\left(\left|f^{\prime}(x)\right|^{q} N_{1}+\left|f^{\prime}(a)\right|^{q} N_{2}\right)^{\frac{1}{q}} \\
& \quad+\frac{\left(b^{\rho}-x^{\rho}\right)^{\alpha+1}}{b-a} M^{\frac{1}{r}}(b)\left(\left|f^{\prime}(x)\right|^{q} N_{1}+\left|f^{\prime}(b)\right|^{q} N_{2}\right)^{\frac{1}{q}},
\end{aligned}
$$

where

$$
\begin{aligned}
& M(a)=\frac{\rho(a x)^{-\rho r}\left(-a^{\rho+r} x^{\rho r}+a^{\rho r} x^{\rho+r}\right)}{(\rho(1-r)+r)\left(x^{\rho}-a^{\rho}\right)}, \\
& M(b)=\frac{\rho(b x)^{-\rho r}\left(-b^{\rho+r} x^{\rho r}+b^{\rho r} x^{\rho+r}\right)}{(\rho(1-r)+r)\left(x^{\rho}-a^{\rho}\right)}, \\
& N_{1}=\frac{\Gamma(1+q) \Gamma\left(\frac{2+\alpha}{\alpha}\right)}{2 \Gamma\left(1+q+\frac{2}{\alpha}\right)}, \\
& N_{2}=\frac{\Gamma(1+q)}{2}\left(\frac{2 \Gamma\left(1+\frac{1}{\alpha}\right)}{\Gamma\left(1+q+\frac{1}{\alpha}\right)}-\frac{\Gamma\left(1+\frac{2}{\alpha}\right)}{\Gamma\left(1+q+\frac{2}{\alpha}\right)}\right),
\end{aligned}
$$

and for all $x \in(a, b], \alpha>0, \rho>0, r>1, q>1, \frac{1}{r}+\frac{1}{q}=1, r \neq \frac{\rho}{\rho-1}$. 
Proof Using Lemma 2.1 and the properties of the absolute value we get

$$
\begin{aligned}
& \left|T_{f}(\alpha, \rho ; a, x, b)\right| \\
& \leq \frac{\left(x^{\rho}-a^{\rho}\right)^{\alpha+1}}{b-a} \int_{0}^{1} \frac{\left(1-t^{\alpha}\right)\left|f^{\prime}\left(\left[t x^{\rho}+(1-t) a^{\rho}\right]^{\frac{1}{\rho}}\right)\right|}{\left(t x^{\rho}+(1-t) a^{\rho}\right)^{1-\frac{1}{\rho}}} d t \\
& \quad+\frac{\left(b^{\rho}-x^{\rho}\right)^{\alpha+1}}{b-a} \int_{0}^{1} \frac{\left(1-t^{\alpha}\right)\left|f^{\prime}\left(\left[t x^{\rho}+(1-t) b^{\rho}\right]^{\frac{1}{\rho}}\right)\right|}{\left(t x^{\rho}+(1-t) b^{\rho}\right)^{1-\frac{1}{\rho}}} d t .
\end{aligned}
$$

By using the Hölder inequality we have

$$
\begin{aligned}
\left|T_{f}(\alpha, \rho ; a, x, b)\right| \leq & \frac{\left(x^{\rho}-a^{\rho}\right)^{\alpha+1}}{b-a}\left(\int_{0}^{1}\left(\left(t x^{\rho}+(1-t) a^{\rho}\right)^{\frac{1}{\rho}-1}\right)^{r} d t\right)^{\frac{1}{r}} \\
& \times\left(\int_{0}^{1}\left(1-t^{\alpha}\right)^{q}\left|f^{\prime}\left(\left[t x^{\rho}+(1-t) a^{\rho}\right]^{\frac{1}{\rho}}\right)\right|^{q} d t\right)^{\frac{1}{q}} \\
& +\frac{\left(b^{\rho}-x^{\rho}\right)^{\alpha+1}}{b-a}\left(\int_{0}^{1}\left(\left(t x^{\rho}+(1-t) b^{\rho}\right)^{\frac{1}{\rho}-1}\right)^{r} d t\right)^{\frac{1}{r}} \\
& \times\left(\int_{0}^{1}\left(1-t^{\alpha}\right)^{q}\left|f^{\prime}\left(\left[t x^{\rho}+(1-t) b^{\rho}\right]^{\frac{1}{\rho}}\right)\right|^{q} d t\right)^{\frac{1}{q}} .
\end{aligned}
$$

Since $\left|f^{\prime}\right|^{q}$ is $\rho$-convex on $I$ we get

$$
\begin{aligned}
\left|T_{f}(\alpha, \rho ; a, x, b)\right| \leq & \frac{\left(x^{\rho}-a^{\rho}\right)^{\alpha+1}}{b-a}\left(\int_{0}^{1}\left(\left(t x^{\rho}+(1-t) a^{\rho}\right)^{\frac{1}{\rho}-1}\right)^{r} d t\right)^{\frac{1}{r}} \\
& \times\left(\int_{0}^{1}\left(1-t^{\alpha}\right)^{q} t\left|f^{\prime}(x)\right|^{q} d t+\int_{0}^{1}\left(1-t^{\alpha}\right)^{q}(1-t)\left|f^{\prime}(a)\right|^{q} d t\right)^{\frac{1}{q}} \\
& +\frac{\left(b^{\rho}-x^{\rho}\right)^{\alpha+1}}{b-a}\left(\int_{0}^{1}\left(\left(t x^{\rho}+(1-t) b^{\rho}\right)^{\frac{1}{\rho}-1}\right)^{r} d t\right)^{\frac{1}{r}} \\
& \times\left(\int_{0}^{1}\left(1-t^{\alpha}\right)^{q} t\left|f^{\prime}(x)\right|^{q} d t+\int_{0}^{1}\left(1-t^{\alpha}\right)^{q}(1-t)\left|f^{\prime}(b)\right|^{q} d t\right)^{\frac{1}{q}}
\end{aligned}
$$

With simple calculation we get

$$
\begin{aligned}
& M(a)=\int_{0}^{1}\left(\left(t x^{\rho}+(1-t) a^{\rho}\right)^{\frac{1}{\rho}-1}\right)^{r} d t \\
& M(b)=\int_{0}^{1}\left(\left(t x^{\rho}+(1-t) b^{\rho}\right)^{\frac{1}{\rho}-1}\right)^{r} d t \\
& N_{1}=\int_{0}^{1}\left(1-t^{\alpha}\right)^{q} t d t, \text { and } N_{2}=\int_{0}^{1}\left(1-t^{\alpha}\right)^{q}(1-t) d t
\end{aligned}
$$

where $M(a), M(b), N_{1}$ and $N_{2}$ are defined as in (36), (37), (38), and (39), respectively. So we get the desired result.

Theorem 2.3 Let $f$ be defined from an interval I which consists of positive real numbers to $\mathbb{R}$ as a differentiable mapping on $I^{\circ}$ and $a, b \in I$ with $a<b$ such that $f^{\prime} \in L[a, b]$. If $\left|f^{\prime}\right|^{q}$ 
is $\rho$-convex on I we have

$$
\begin{aligned}
& \left|T_{f}(\alpha, \rho ; a, x, b)\right| \\
& \quad \leq \frac{\left(x^{\rho}-a^{\rho}\right)^{\alpha+1}}{b-a}\left(\frac{\rho(x-a)}{x^{\rho}-a^{\rho}}\right)^{1-\frac{1}{q}}\left(\left|f^{\prime}(x)\right|^{q} K(a)+\left|f^{\prime}(a)\right|^{q} L(a)\right)^{\frac{1}{q}} \\
& \quad+\frac{\left(b^{\rho}-x^{\rho}\right)^{\alpha+1}}{b-a}\left(\frac{\rho(b-x)}{b^{\rho}-x^{\rho}}\right)^{1-\frac{1}{q}}\left(\left|f^{\prime}(x)\right|^{q} K(b)+\left|f^{\prime}(a)\right|^{q} L(b)\right)^{\frac{1}{q}},
\end{aligned}
$$

where $K(a), K(b), L(a)$ and $L(b)$ are defined as in Theorem 2.1 and for all $x \in\left(a, 2^{\frac{1}{\rho}} a\right)$ (if $2^{\frac{1}{\rho}} a<b$, otherwise $\left.x \in(a, b)\right), \alpha>0, \rho>1, q>1, \frac{1}{\rho}+\frac{1}{q}=1$.

Proof Using Lemma 2.1 and the properties of the absolute value we get

$$
\begin{aligned}
\left|T_{f}(\alpha, \rho ; a, x, b)\right| \leq & \frac{\left(x^{\rho}-a^{\rho}\right)^{\alpha+1}}{b-a} \int_{0}^{1} \frac{\left(1-t^{\alpha}\right)\left|f^{\prime}\left(\left[t x^{\rho}+(1-t) a^{\rho}\right]^{\frac{1}{\rho}}\right)\right|}{\left(t x^{\rho}+(1-t) a^{\rho}\right)^{1-\frac{1}{\rho}}} d t \\
& +\frac{\left(b^{\rho}-x^{\rho}\right)^{\alpha+1}}{b-a} \int_{0}^{1} \frac{\left(1-t^{\alpha}\right)\left|f^{\prime}\left(\left[t x^{\rho}+(1-t) b^{\rho}\right]^{\frac{1}{\rho}}\right)\right|}{\left(t x^{\rho}+(1-t) b^{\rho}\right)^{1-\frac{1}{\rho}}} d t .
\end{aligned}
$$

With the help of the power-mean inequality we have

$$
\begin{aligned}
\left|T_{f}(\alpha, \rho ; a, x, b)\right| \leq & \frac{\left(x^{\rho}-a^{\rho}\right)^{\alpha+1}}{b-a}\left(\int_{0}^{1} \frac{1-t^{\alpha}}{\left(t x^{\rho}+(1-t) a^{\rho}\right)^{1-\frac{1}{\rho}}} d t\right)^{1-\frac{1}{q}} \\
& \times\left(\int_{0}^{1} \frac{\left(1-t^{\alpha}\right)\left|f^{\prime}\left(\left[t x^{\rho}+(1-t) a^{\rho}\right]^{\frac{1}{\rho}}\right)\right|^{q}}{\left(t x^{\rho}+(1-t) a^{\rho}\right)^{1-\frac{1}{\rho}}} d t\right)^{\frac{1}{q}} \\
& +\frac{\left(b^{\rho}-x^{\rho}\right)^{\alpha+1}}{b-a}\left(\int_{0}^{1} \frac{1-t^{\alpha}}{\left(t x^{\rho}+(1-t) b^{\rho}\right)^{1-\frac{1}{\rho}}} d t\right)^{1-\frac{1}{q}} \\
& \times \int_{0}^{1}\left(\frac{\left(1-t^{\alpha}\right)\left|f^{\prime}\left(\left[t x^{\rho}+(1-t) b^{\rho}\right]^{\frac{1}{\rho}}\right)\right|^{q}}{\left(t x^{\rho}+(1-t) b^{\rho}\right)^{1-\frac{1}{\rho}}} d t\right)^{\frac{1}{q}}
\end{aligned}
$$

and by using the $\rho$-convexity of $\left|f^{\prime}\right|^{q}$, then using the Hölder inequality we have

$$
\begin{aligned}
T_{f}(\alpha, \rho ; a, x, b) \leq & \frac{\left(x^{\rho}-a^{\rho}\right)^{\alpha+1}}{b-a}\left(\int_{0}^{1} \frac{1-t^{\alpha}}{\left(t x^{\rho}+(1-t) a^{\rho}\right)^{1-\frac{1}{\rho}}} d t\right)^{1-\frac{1}{q}} \\
& \times\left\{\begin{array}{c}
\left|f^{\prime}(x)\right|^{q} \int_{0}^{1}\left(t-t^{\alpha+1}\right)\left(t x^{\rho}+(1-t) b^{\rho}\right)^{\frac{1}{\rho}-1} d t \\
\left|f^{\prime}(a)\right|^{q} \int_{0}^{1}(1-t)\left(1-t^{\alpha}\right)\left(t x^{\rho}+(1-t) a^{\rho}\right)^{\frac{1}{\rho}-1} d t
\end{array}\right\}^{\frac{1}{q}} \\
& +\frac{\left(b^{\rho}-x^{\rho}\right)^{\alpha+1}}{b-a}\left(\int_{0}^{1} \frac{1-t^{\alpha}}{\left(t x^{\rho}+(1-t) a^{\rho}\right)^{1-\frac{1}{\rho}}} d t\right)^{1-\frac{1}{q}} \\
& \times\left\{\begin{array}{c}
\left|f^{\prime}(x)\right|^{q} \int_{0}^{1}\left(t-t^{\alpha+1}\right)\left(t x^{\rho}+(1-t) b^{\rho}\right)^{\frac{1}{\rho}-1} d t \\
\left|f^{\prime}(b)\right|^{q} \int_{0}^{1}(1-t)\left(1-t^{\alpha}\right)\left(t x^{\rho}+(1-t) b^{\rho}\right)^{\frac{1}{\rho}-1} d t
\end{array}\right\}^{\frac{1}{q}} \\
\leq & \frac{\left(x^{\rho}-a^{\rho}\right)^{\alpha+1}}{b-a}\left(\int_{0}^{1} \frac{\left(1-t^{\alpha}\right)}{\left(t x^{\rho}+(1-t) a^{\rho}\right)^{1-\frac{1}{\rho}}} d t\right)^{1-\frac{1}{q}}
\end{aligned}
$$




$$
\begin{aligned}
& \times\left\{\begin{array}{c}
\left|f^{\prime}(x)\right|^{q} \int_{0}^{1}\left(t-t^{\alpha+1}\right)\left(t x^{\rho}+(1-t) b^{\rho}\right)^{\frac{1}{\rho}-1} d t \\
\left.f^{\prime}(a)\right|^{q}\left(\int_{0}^{1}(1-t)^{\rho}\left(t x^{\rho}+(1-t) a^{\rho}\right)^{1-\rho} d t\right)^{\frac{1}{\rho}}\left(\int_{0}^{1}\left(1-t^{\alpha}\right)^{q} d t\right)^{\frac{1}{q}}
\end{array}\right\}^{\frac{1}{q}} \\
& +\frac{\left(b^{\rho}-x^{\rho}\right)^{\alpha+1}}{b-a}\left(\int_{0}^{1} \frac{1-t^{\alpha}}{\left(t x^{\rho}+(1-t) a^{\rho}\right)^{1-\frac{1}{\rho}}} d t\right)^{1-\frac{1}{q}} \\
& \times\left\{\begin{array}{c}
\left|f^{\prime}(x)\right|^{q} \int_{0}^{1}\left(t-t^{\alpha+1}\right)\left(t x^{\rho}+(1-t) b^{\rho}\right)^{\frac{1}{\rho}-1} d t \\
\left|f^{\prime}(b)\right|^{q}\left(\int_{0}^{1}(1-t)^{\rho}\left(t x^{\rho}+(1-t) b^{\rho}\right)^{1-\rho} d t\right)^{\frac{1}{\rho}}\left(\int_{0}^{1}\left(1-t^{\alpha}\right)^{q} d t\right)^{\frac{1}{q}}
\end{array}\right\}^{\frac{1}{q}} .
\end{aligned}
$$

By simple computation we get

$$
\begin{aligned}
\int_{0}^{1} \frac{1-t^{\alpha}}{\left(t x^{\rho}+(1-t) a^{\rho}\right)^{1-\frac{1}{\rho}}} d t & =\frac{\rho}{x^{\rho}-a^{\rho}}\left[(x-a)-\int_{a}^{x}\left(\frac{u^{\rho}-a^{\rho}}{x^{\rho}-a^{\rho}}\right)^{\alpha} d u\right] \\
& <\frac{\rho(x-a)}{x^{\rho}-a^{\rho}}, \\
\int_{0}^{1} \frac{1-t^{\alpha}}{\left(t x^{\rho}+(1-t) b^{\rho}\right)^{1-\frac{1}{\rho}}} d t & =\frac{\rho}{b^{\rho}-x^{\rho}}\left[(b-x)-\int_{x}^{b}\left(\frac{b^{\rho}-u^{\rho}}{b^{\rho}-x^{\rho}}\right)^{\alpha} d u\right] \\
& <\frac{\rho(b-x)}{b^{\rho}-x^{\rho}},
\end{aligned}
$$

and

$$
\begin{aligned}
& K(a)=\int_{0}^{1}\left(t-t^{\alpha+1}\right)\left(t x^{\rho}+(1-t) a^{\rho}\right)^{\frac{1}{\rho}-1} d t, \\
& K(b)=\int_{0}^{1}\left(t-t^{\alpha+1}\right)\left(t x^{\rho}+(1-t) b^{\rho}\right)^{\frac{1}{\rho}-1} d t, \\
& L(a)=\left(\int_{0}^{1} \frac{(1-t)^{\rho}}{\left(t x^{\rho}+(1-t) a^{\rho}\right)^{\rho-1}} d t\right)^{\frac{1}{\rho}}\left(\int_{0}^{1}\left(1-t^{\alpha}\right)^{q} d t\right)^{\frac{1}{q}}, \\
& L(b)=\left(\int_{0}^{1} \frac{(1-t)^{\rho}}{\left(t x^{\rho}+(1-t) b^{\rho}\right)^{\rho-1}} d t\right)^{\frac{1}{\rho}}\left(\int_{0}^{1}\left(1-t^{\alpha}\right)^{q} d t\right)^{\frac{1}{q}},
\end{aligned}
$$

where $K(a), K(b), L(a)$ and $L(b)$ are defined as in (26), (27), (28) and (29), respectively. So the proof is completed.

\section{Acknowledgements}

The authors would like to thank the anonymous referees and the editor for their constructive suggestions on improving the presentation of the paper.

\section{Funding}

There is no funding for this work.

Availability of data and materials

Not applicable.

Competing interests

The authors declare that they have no competing interests. 


\section{Author details}

${ }^{1}$ Department of Mathematics, Education Faculty, Ağrı Ibrahim Çeçen University, Ağrı, Turkey. ${ }^{2}$ Institute of Science, Ağrı İbrahim Çeçen University, Ağrı, Turkey. ${ }^{3}$ Department of Mathematics, Faculty of Science and Arts, Ordu University, Ordu, Turkey.

\section{Publisher's Note}

Springer Nature remains neutral with regard to jurisdictional claims in published maps and institutional affiliations.

Received: 4 March 2020 Accepted: 22 May 2020 Published online: 03 June 2020

\section{References}

1. Erdélyi, A., Magnus, W., Oberhettinger, F., Tricomi, F.: Higher Transcendental Functions, Vol. I-III. Krieger, Melbourne (1981)

2. Guessab, A., Schmeisser, G.: Sharp integral inequalities of the Hermite-Hadamard type. J. Approx. Theory 115(2), 260-288 (2002)

3. İşcan, I.: Ostrowski type inequalities for p-convex functions. New Trends Math. Sci. 3, 140-150 (2016). https://doi.org/10.20852/ntmsci.2016318838

4. Katugampola, U.N.: New approach to a generalized fractional integral. Appl. Math. Comput. 218(3), 860-865 (2011). https://doi.org/10.1016/j.amc.2011.03.062

5. Katugampola, U.N.: New approach to generalized fractional derivatives. Bull. Math. Anal. Appl. 6(4), 1-15 (2014)

6. Kavurmacl, H., Avcı, M., Özdemir, M.E.: New inequalities of Hermite-Hadamard type for convex functions with applications. J. Inequal. Appl. 2011, 86 (2011). https://doi.org/10.1186/1029-242X-2011-86

7. Kavurmacl, H., Avcı, M., Özdemir, M.E.: Hermite-Hadamard type inequalities for s-convex and s-concave functions via fractional integrals (2012) arXiv:1202.0380v1

8. Kilbas, A.A.: Hadamard-type fractional calculus. J. Korean Math. Soc. 38(6), 1191-1204 (2001)

9. Kilbas, A.A., Srivastava, H.M., Trujillo, J.J.: Theory and Applications of Fractional Differential Equations. North-Holland Mathematics Studies (2006)

10. Qiu, K., Wang, J.R.: A fractional integral identity and its application to fractional Hermite-Hadamard type inequalities. J. Interdiscip. Math. 21(1), 1-16 (2018). https://doi.org/10.1080/09720502.2017.1400795

11. Shiba, N., Takayanagi, T.: Volume law for the entanglement entropy in non-local QFTs. J. High Energy Phys. 2(33), 1 (2014). https://doi.org/10.1007/JHEP02(2014)033

12. Usta, F., Sarıkaya, M.Z.: Some improvements of conformable fractional integral inequalities. Int. J. Anal. Appl. 14(2), $162-166$ (2017)

13. Zhang, K.S., Wan, J.P.: p-Convex functions and their properties. Pure Appl. Math. 23(1), 130-133 (2007)

\section{Submit your manuscript to a SpringerOpen ${ }^{\circ}$ journal and benefit from:}

- Convenient online submission

- Rigorous peer review

Open access: articles freely available online

- High visibility within the field

Retaining the copyright to your article

Submit your next manuscript at $\boldsymbol{\triangleright}$ springeropen.com 